\title{
ANÁLISIS ESTADÍSTICO DE LOS PARÁMETROS ACK_TIMEOUT, CTS TIMEOUT Y SLOT_TIME EN ENLACES DE LARGA DISTANCIA 802.11g
}

\author{
STATISTICAL ANALYSIS OF PARAMETERS ACK_TIMEOUT, CTS_TIMEOUT AND \\ SLOT_TIME LINKS IN LONG DISTANCE $802.11 \mathrm{~g}$
}

Eliana Grisales Zuluaga ${ }^{2}$, Héctor Fabio Bermúdez Orozco. ${ }^{1,2}$, Evelio Astaiza Hoyos. ${ }^{1,2}$

1. Grupo de Investigación en telecomunicaciones GITUQ.
2 Programa de Ingeniería Electrónica, Universidad del Quindío. Armenia, Colombia
egrisalesz@uqvirtual.edu.co, hfbermudez@uniquindio.edu.co,
eastaiza@uniquindio.edu.co.
*Correspondencia del autor. Programa de Ingeniería Electrónica, Universidad del Quindío. Armenia, Colombia.
egrisalesz@uqvirtual.edu.co

\section{RESUMEN}

El presente proyecto plantea como fin utilizar el protocolo de comunicaciones $802.11 \mathrm{~g}$, teniendo en cuenta sus limitaciones, para mejorar la capacidad de transmisión de datos variando parámetros constituyentes de esta tecnología como lo son el CTS/RTS, SLOT Time y ACK Time Out en función tanto de la capacidad como de la velocidad de transmisión. Para esto el reciente trabajo consta de la definición de cuatro escenarios donde posteriormente se realizaron las mediciones de tasa de transferencia, las muestras obtenidas en cada escenario se analizaron por medio de la prueba estadística de Kolmogorov-Smirnov y se encontraron las distribuciones de probabilidad de los datos tabulados con lo que se consigue una interpretación más sencilla de estos y así se adquiere la mayor cantidad posible de información que permite identificar los valores que maximizan la tasa de transferencia.

Se observa que en su mayoría las distribuciones de datos de cada parámetro son de tipo uniforme, por ello, los valores que alcanzan mayor tasa de transferencia presentan un amplio rango alrededor de su media, también se observa que el ajuste del parámetro ACK Time Out resulta significativo en cuanto al desempeño puesto que su variación puede producir los puntos más críticos del enlace.

El ajuste adecuado de estos parámetros también permite comprobar que IEEE802.11g es apto al aplicarse a largas distancias, especialmente a zonas rurales aisladas con baja densidad de población, ya que se consiguieron velocidades hasta de 19 Mbps que resultan adecuado para establecer enlaces de varios kilómetros con buena calidad.

Palabras claves: ACK Time Out, CTS Time Out, Slot Time, IEEE802.11g, Enlace, Kolmogorov-Smirnov. 


\begin{abstract}
This project proposes to use as end the communication protocol $802.11 \mathrm{~g}$, considering its limitations, to improve data transmission capability constituents varying technology parameters such as the CTS/RTS, and ACK Time out SLOTTIME according to both the capacity and transmission speed. For this recent work consisted of defining four scenaries where later measurements transfer rate were performed, the samples obtained in each scenario were analyzed by means of statistical Kolmogorov- Smirnov and probability distributions of found tabular data with a simpler interpretation of these forces and thus the maximum amount of information that identifies the values that maximize the transfer rate is acquired.

It is observed that most of the data distributions of each parameter are of uniform type, therefore, reaching values higher transfer exhibit a wide range around the average, which shows that the parameter setting ACK Time Out is significant in terms of performance since its variation can produce the most critical points of the connection.

Proper adjustment of these parameters also allows checking that IEEE802.11g is suitable when applied to long distances, especially in remote rural areas with low population density, as speeds got up to 19 Mbps that are suitable for linking several kilometers with good quality.
\end{abstract}

Keywords: ACK Time Out, CTS Time Out, Slot Time, IEEE802.11g, Link, Kolmogorov-Smirnov.

\section{INTRODUCCIÓN}

Es bien sabido que las comunidades aisladas, ubicadas en zonas geográficas de baja densidad de población, y que generalmente presentan bajos ingresos económicos carecen de algunos servicios como lo es el de comunicaciones, esto, debido a que para los operadores y prestadores de servicios de comunicaciones no resulta atractivo y rentable invertir en infraestructura además de funcionamiento para la prestación de servicios a estas comunidades; la motivación de este trabajo se basa en aprovechar una de las aplicaciones de las WLAN no tan importantes como lo es el establecimiento de enlaces de larga distancia para brindar cobertura y servicios a este tipo de comunidades; con esto se busca abrir las posibilidades de mejoramiento de las condiciones de salud y educación de la población en la medida de implementación de soluciones de Telemedicina y Teleducación a través de los medios de comunicaciones aprovechables, generando grandes impactos sociales en el mejoramiento de la calidad de vida de la población beneficiada por las muchas aplicaciones y servicios de comunicaciones adicionales que pueden ser considerados. El desarrollo de este proyecto genera un alto impacto tanto desde la perspectiva social, económica y educativa, ya que propende por el mejoramiento de la infraestructura de comunicaciones inalámbricas que operan en bandas de libre uso y que permitan generar este tipo de soluciones; pero para ello es importante conocer el estándar de la IEEE 802.11 (1) y tener claro que aunque no se concibió para redes extensas, sus indudables ventajas de costo, uso de frecuencias libres de licencia y gran ancho de banda, lo hacen de interés para este tipo de aplicaciones, más aún si se conocen sus limitaciones y se obtienen los suficientes parámetros que permitan extraer información acerca de cómo mejorar su desempeño.

El método que se plantea para conseguir lo anterior se basa en realizar la evaluación del desempeño de la máxima capacidad alcanzada en enlaces de larga distancia $802.11 \mathrm{G}$ por medio de la variación de los parámetros de la capa de enlace como lo son CTS Time Out, SLOT Time y ACK Time Out, para determinar de una manera experimental, cuáles de esas variación generan las tasas de transferencia de información más elevadas, en función de la distancia del enlace y de las características de propagación de la zona sobre la cual operan los enlaces.

Por lo tanto, en el desarrollo de este proyecto, se establecieron cuatro escenarios en los cuales la topología del medio cambiaba, en cada una de las cuatro pruebas de campo se midieron los parámetros en función de la distancia y las características de propagación de la zona, esto, con el fin de encontrar patrones que hicieran que el análisis de la datos obtenidos fuera un poco coherente y poder determinar si la influencia de las variaciones geográficas afectaban de forma significativa la velocidad obtenida en la transferencia de información.

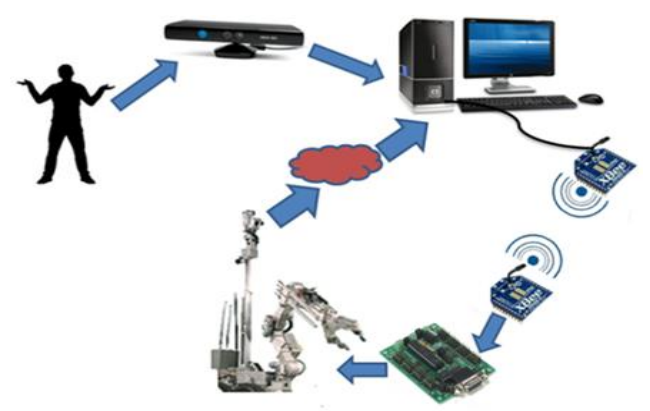

Figura 1. Propuesta del sistema implementado 
Definición de los parámetros medidos:

Para definir los parámetros estudiados, se toma entonces el estándar IEEE802.11 y se determinan las tramas de control ACK Time Out, RTS Time Out y Slottime las cuales ayudan con la entrega de información entre las estaciones.

\section{- Trama Request to Send (RTS)}

El término anterior es una abreviatura de petición de envío. Si un dispositivo inalámbrico quiere comunicarse con otro, éste transmite una unidad de datos, conocida como RTS al destinatario y espera a que éste reconozca que está listo. La estación envía una trama RTS para iniciar el diálogo de comienzo de transmisión de una trama. (2)

El formato para esta trama es el siguiente:

RA (Receiver Address): es la dirección de la estación destino de la trama pendiente, de datos o administración, que se requiere transferir posterior al intercambio RTS/CTS.

TA (Transmitter Address): es la dirección de la esta-

\begin{tabular}{|c|c|c|c|c|}
\hline Octetos: & 2 & 6 & 6 & 4 \\
\hline Control de trama & Duración & RA & TA & FCS \\
\hline
\end{tabular}

Figura 1. Formato de la trama RTS.

ción transmisora de la trama RTS

Duration: es el tiempo, en microsegundos, requerido para transmitir la trama de datos o administración pendiente, más un CTS, una trama ACK, y tres intervalos SIFS. Si la duración calculada incluye una fracción de microsegundo, el valor es redondeado al entero inmediatamente superior. (3)

\section{- Trama Clear to send (CTS)}

Clear to send es una función que permite al accespoint controlar el uso del medio de las estaciones. Cuando se habla de CTS es imposible dejar de hablar de RTS.

El adaptador activa RTS/CTS, cuando esto sucede se envía una trama RTS al acces-point antes de iniciar el envío de una trama de datos. Así el acces-point responde con una trama CTS que indica que hay vía libre en el medio para enviar la trama de datos.

El uso de esta herramienta tiene una connotación mayor y es que evita colisiones entre nodos ocultos que no se ven entre sí.

Su formato para esta trama es el siguiente:

- Tramas Acknowledgement (ACK)

El envío de las tramas ACK tienen como objetivo confirmar la recepción de una trama. En caso de no llegar la trama ACK el emisor vuelve a enviar la trama de datos. (2)

Formato de la trama:

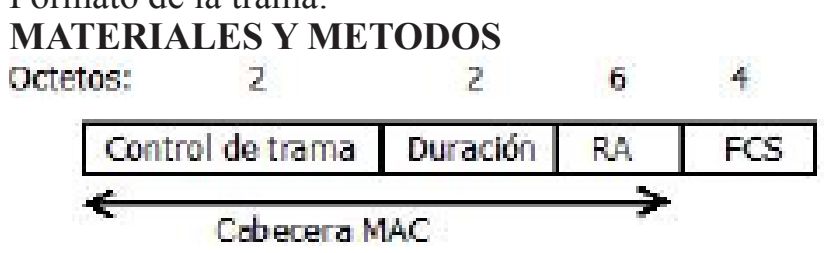

Figura 2. Formato CTS/RTS.

El procedimiento inicialmente se basó en utilizar fuentes como la observación y la investigación por medio de enlaces web para consolidar las bases teóricas para el planteamiento del presente trabajo, el paso a seguir abarcaba el establecimiento de los respectivos enlaces para la toma de datos, de esta manera se hace posible

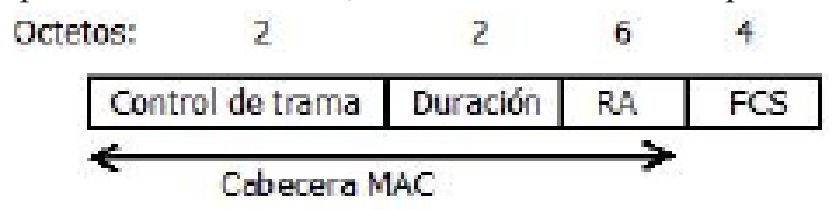

Figura 3. Formato de la trama ACK.

tratar dichos datos y sustentar los resultados por medio de trabajos anteriores y textos referentes al tema, entablando los argumentos necesarios. (4)

El objetivo de esta investigación es realizar diferentes enlaces inalámbricos, es decir implementar redes inalámbricas (conexión de nodos sin necesidad de una conexión física) en diferentes zonas de cobertura (rural, urbana, mixta) con el fin de evaluar el desempeño basado en capacidad de la tecnología $802.11 \mathrm{~g}$ a partir de mediciones de la tasa máxima de transferencia de información en enlaces de larga distancia, obtenida bajo condiciones estándar de funcionamiento y bajo condiciones de modificación en los valores de los parámetros slot time, ACK time-out y CTS time-out.

Equipos físicos y software libre han sido las herramientas utilizadas en esta investigación; para entablar los enlaces punto a punto se escogieron los acces point Ubiquiti Power Station conectados a antenas rejilla de referencia WLG-5775-27 y los Ubiquiti Power Station 2 de referencia PS2-17D los cuales tienen las antenas integradas con características de doble polarización.

Estos equipos poseen características similares; por una parte estas estaciones son caracterizadas por estar basadas en la arquitectura 802.11 la cual es versátil y abierta basada en plataformas inalámbricas que pueden ser usadas como AP, Bridge, estación PMP, clien- 
te de máscara de red y en muchas otras aplicaciones de desarrollo; y por otra parte, las antenas utilizadas son de carácter directivo es decir, que concentran toda la energía de radiación en una dirección preferente, con una ganancia muy importante en esa dirección a

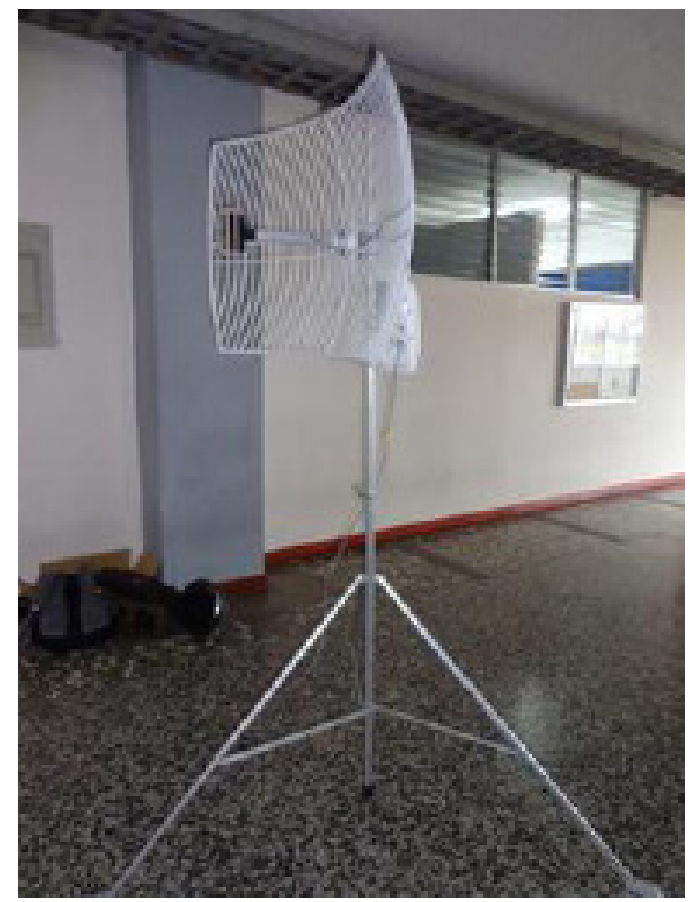

Figura 4. Ubiquiti Power Station conectado a una antena rejilla.
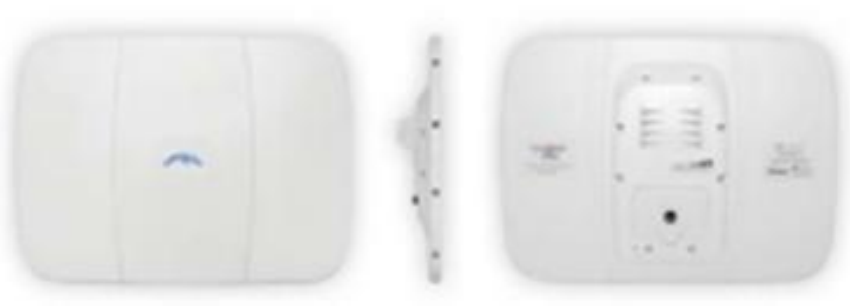

Figura 5. Ubiquiti Power Station 2 PS2-17D, con antenas integradas.

costa de disminuir las otras direcciones.

Se decidió plantear cuatro enlaces diferentes, es decir, realizar redes inalámbricas en cuatro escenarios con diversas características y por medio de ellas observar los cambios en la tasa de envío de información de datos, esto teniendo en cuenta los cambios en la topografía del terreno. Es importante destacar que la distancia en las comunicaciones puede ser un factor no sólo importante, sino definitivo para el análisis de su comportamiento, por ello se utilizó el GPS Garmin Map 62 S con memoria USB de 2 GBytes; este instrumento determinó las coordenadas específicas de los sitios, teniendo en cuenta la información suministrada por éste, se introduce la utilización del software libre

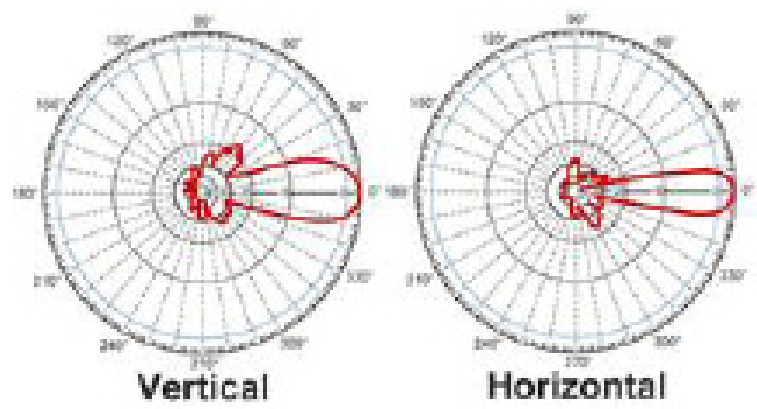

Figura 6. Patrón característico de las antenas directivas.

"Radio Mobile", el cual puede determinar la distancia de los enlaces, simular las comunicaciones y dibujar la elevación en los mapas usando los datos SRTM descargados desde Space Shuttle Radar Terrain Mapping Mission, con la posibilidad de agregar los mapas de rutas y autopistas simultáneamente a los relieves del contorno, obviamente junto a las curvas de nivel.

Como prioridad se deseaba trabajar en condiciones ideales, es por esto que se optó por usar "inSSIDer", el cual es una herramienta (software libre) que permitirá buscar redes inalámbricas en la zona en la cual se encuentra el investigador y controlar, de un modo gráfico, la intensidad de sus señales. Concretamente, con inSSIDer se pueden detectar todas las redes inalámbricas que ofrecen cobertura en la zona debidamente estudiada o analizada y listar en pantalla todos sus detalles: SSID, dirección MAC, canal, RSSI, tipo de red y seguridad, velocidad e intensidad de la señal.

Teniendo en cuenta lo anterior, es de vital importancia destacar que, cada uno de los equipos de cómputo (estación base y estación receptora) debían tener una configuración determinada para lograr que el enlace se entablara correctamente, acciones como desactivar el firewall, el proxy, el antivirus y configurar la dirección del equipo son factores determinantes que harán que un enlace sea exitoso o no.

Tras tener configurados cada uno de los equipos de cómputo que cumplen con el papel de base y subordinado, destinados al estudio del desempeño de la máxima capacidad alcanzada en enlaces de larga distancia, y de los dispositivos nombrados anteriormente (acces point Ubiquiti Power Station), se utiliza un software destinado para la medición de las tasas de transferencia de información denominado "LAN Speed Test"; éste es una herramienta que tiene como fin, medir la velocidad de la transferencia de archivos pertenecientes al disco duro, unidad USB (desde un equipo base a un equipo subordinado), y la Red de Área Local (LAN) (por cable e inalámbrica). Esto, lo hace mediante la construcción de un archivo en la memoria del dispositivo que desea enviar los datos, 
transfiriéndolos a la carpeta destino (sin efectos de ventanas archivo caché), manteniendo un registro de las veces, y luego realiza los cálculos necesarios para tener el dato correcto de la transferencia de archivos tanto promedio, como máximo y mínimo. Es importante destacar que el programa forma paquetes de tamaños de hasta $9 \mathrm{MB}$ y el número de los mismos que se desea transferir.

Para medir la velocidad de la transferencia de archivos en el presente trabajo, se configuró el LAN Speed Test en cada una de las medidas con un número de paquetes igual a cinco y cada uno con tamaño de $1 \mathrm{MB}$, entonces, mediante la construcción de un archivo con estas características en la memoria del dispositivo de destino, se obtenían los datos permitidos por la interfaz del software, posteriormente, de manera manual y con la ayuda de la herramienta Microsoft Excel 2010, se registraban estos datos en tablas.

Debido a que la información que se tiene en tablas y gráficas no presenta un comportamiento que permita visualizar los valores que producen la máxima capacidad, es necesario modificar la forma de representación de dichos datos y presentarla como tablas de frecuencia, con la finalidad de realizar la prueba de Kolmogorov-Smirnov para encontrar el tipo de distribución de probabilidad de los datos obtenidos; esta prueba permite medir el grado de concordancia existente entre la distribución de un conjunto de datos y una distribución teórica especificada.

Es posible utilizar la prueba de bondad de Kolmogorov-Smirnov ya que trabaja con la distribución de probabilidad acumulada. La metodología es la siguiente:

1. Se colocan los $\mathrm{n}$ datos históricos en una tabla de frecuencia observada (F.O.) con $m=$ raíz(n) intervalos. Se obtiene la frecuencia observada en cada intervalo i (FOi) como la cantidad de datos en cada intervalo de tamaño $\mathrm{T}$.

Se calcula la media y la varianza de los datos como:

2. Se propone una distribución de probabilidad de acuerdo con la forma de la tabla de frecuencias obtenida en el paso 1, graficando un histograma de frecuencia observada versus intervalos.

3. Se calcula la frecuencia observada acumulada (FOA) para cada intervalo como:

4. Se calcula la probabilidad observada acumulada (POA) para cada intervalo como

5. Con la distribución propuesta se calcula la probabilidad esperada para cada uno de los intervalos como

Para distribuciones continuas

Para distribuciones discretas:

6. Se calcula el estimador de la máxima diferencia DM como

7. Se obtiene el correspondiente valor de la distribu- ción de Kolmogorov-Smirnov (D) para $\mathrm{n}$ grados de libertad y a un nivel de confiabilidad de 1-alfa

$$
T=\frac{>\text { dato }-<\text { dato }}{m}(1)
$$

de la tabla de dicha distribución.

$$
\text { Hedia }=\frac{1}{n} \sum_{i=1}^{n} x_{i}(2)
$$

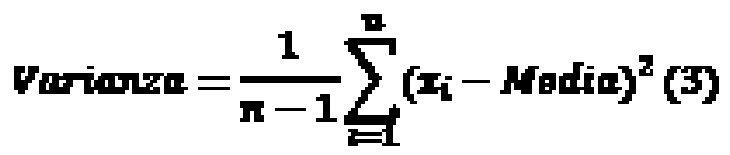

El estimador DM se compara con el valor límite Dn, a-alfa. Si el estimador DM es menor o igual al valor límite, entonces se dice que la hipótesis propuesta se ajusta a la distribución de probabilidad que sigue la información histórica. En caso contrario se debe retornar al punto 2 y realizar nuevamente el proceso. (5)

$$
F O A_{i}=\sum_{i=1}^{i} F O_{n}(4)
$$

$$
\begin{aligned}
& \text { RESULTADOS Y DISCUSIÓN } \\
& \qquad P O A_{i}=\frac{F O A_{i}}{n}(5)
\end{aligned}
$$

Fue significativo tener en cuenta que las limitaciones más importantes detectadas en la planeación de

$$
P E A_{i}=\left[\left.F(x)\right|_{0} ^{L S}\right](6)
$$

Dónde:

$$
F(x)=\int_{D}^{x} f(y) d y(7)
$$

$$
F(x)=\mathbf{X} P(y)(\mathbf{8})
$$

enlaces de larga distancia son varias: la primera y más importante es la necesidad de línea de vista entre emisor y receptor, a lo cual se le da solución aprove-

$$
D M=M a ́ x\left|P E A_{i}-P O A_{i}\right|(9)
$$

chando las condiciones de terrenos que cumplían con las características planeadas, teniendo en cuenta que debe garantizarse la comunicación mirando la primera zona de fresnel, sin embargo entre mayor sea la distancia del enlace hay que tener en cuenta que la cur- 
vatura de la tierra afecta el enlace obligando a elevar las antenas, para este caso no resultó necesario elevar demasiado las antenas ya que los puntos escogidos para su instalación y la distancia de por medio eran favorables; la segunda es la vulnerabilidad de trabajar en la banda de frecuencia libre, ya que por las características del medio usado, es inevitable darse cuenta de qué dispositivos se encuentren en las cercanías de los nuestros, trabajando en esta banda, nos afectarán produciendo interferencias (todas estas interferencias provocan que la red inalámbrica no funcione a su más alto rendimiento), el efecto de otros radioenlaces y diferentes tipos de dispositivos operando en la misma frecuencia pueden llegar a ser perjudiciales, por lo que es importante tener en cuenta los factores dichos anteriormente en el momento de diseñar la red; la tercera es la limitación económica a la hora de garantizar la potencia necesaria, esto se soluciona mediante el uso adecuado de antenas direccionales.

Para la planeación de los enlaces en los cuales se necesitaba realizar las respectivas mediciones de tasa de transferencia, se estudiaron diferentes terrenos; en sí se establecieron cuatro escenarios, en cada uno se utilizó el software radiomobile para hacer la simulación de la comunicación y demostrar que efectivamente dichas comunicaciones existían de un punto a otro.

Con esto, para el primer escenario definido entre la Universidad del Quindío y una casa en el barrio "Modelo" de la ciudad de Armenia, se obtiene una distancia aproximada de $2,39 \mathrm{Km}$; por las características del terreno, las edificaciones y la variación de las calles puede afectar el medio de transmisión de las señales, o sea, influye en la variación de las variables a estudiar y un enlace realizado en una zona rodeada de casas, construcciones y edificios, puede de una u otra manera influir en la velocidad de la tasa de transferencia de archivos.

Para el siguiente escenario, definido entre el cerro "El Castillo" ubicado en el municipio de Calarcá y la "Báscula Tigreros" ubicada en la zona sur de la ciudad de Armenia, el terreno es característico de una zona rural; la línea de vista entre las antenas tanto receptora como transmisora no presentaba ningún obstáculo, teniendo esto claro, se esperaría que este enlace de como resultado una tasa de transferencia de información con muy buena calidad.

Como un tercer escenario el enlace se estableció entre una finca en el municipio de Córdoba y "Comfenalco El Bosque" de la ciudad de Armenia, esta zona se puede denominar como mixta (componente urbano y rural), el cual presenta una distancia de $17,96 \mathrm{~km}$; el conjunto de edificios constituyentes de la zona urbana además de árboles, montañas o demás factores perte- necientes a los accidentes geográficos de una región pueden hacer que la velocidad de la transmisión de archivos cambie debido a los fenómenos de multitrayecto, refracción y difracción que las señales enviadas pueden sufrir.

Un cuarto y último escenario se estableció entre la alcaldía del municipio de Tebaida y una casa en el barrio "Bosque de Pinares" de la ciudad de Armenia, que de igual forma, por las características que presenta el terreno se puede denominar como mixto.

Después de analizar y procesar los valores de tasa de transferencia obtenidos a partir de la variación de los parámetros especificados con anterioridad en el presente informe, se observa que las tasas de transferencia de información alcanzan velocidades hasta de 19 Mbps en el enlace de subida, los datos que se presentan a continuación son el resultado de promediar las tasas máximas de transferencia de información en el enlace de subida y bajada.

Para cada entorno se tienen los siguientes datos más significativos:

Los valores de los parámetros anteriores, aunque a simple vista son los que permiten las tasas de transferencia de información más elevadas, no presentan un comportamiento predecible a medida que cambian, es por ello que se opta por utilizar el método estadístico nombrado anteriormente para ajustar el conjunto de datos obtenidos a una función de distribución continua de probabilidad y con ello obtener resultados de mayor confiabilidad.

Se observa que en su mayoría las distribuciones de datos de cada parámetro son de tipo uniforme, con un nivel de confiabilidad del $95 \%$ en el ajuste a dicha distribución (dato obtenido de comparar los datos con la tabla de Kolmogorov Smirnov), por ello los valores que alcanzan mayor tasa de transferencia presentan un amplio rango alrededor de su media y se puede deducir que es posible mantener determinada tasa con un nivel aceptable de velocidad de transmisión.

Para llevar a cabo el análisis con este método se toman los cuatro escenarios en paralelo, es decir, todas las gráficas resultantes de escritura promedio se organizaron una debajo de otra, y así mismo con las demás mediciones (lectura promedio, escritura/lectura máxima y lectura/escritura mínima), además, en cada una de ellas se calcula la media aritmética de los valores de velocidad de transferencia obtenidos, a partir de 
Tabla 1. Tasa transferencia Barrio Modelo - Universidad del Quindío.

\begin{tabular}{|l|c|}
\hline ACK & Tasa de Transferencia (Mbps) \\
\hline 81 & 10,3836 \\
\hline 141 & 11,0437 \\
\hline 171 & 11,5661 \\
\hline RTS & 14,8249 \\
\hline 2186 & 15,6603 \\
\hline 2106 & 14,4495 \\
\hline 1346 & \\
\hline SLOTTIME & 16,6549 \\
\hline 2346 & 17,059 \\
\hline 2106 & 16,9678 \\
\hline 1946 & \\
\hline
\end{tabular}

Tabla 2. Tasa transferencia Cerro El Castillo - Báscula Tigreros.

\begin{tabular}{|l|c|}
\hline ACK & Tasa de Transferencia (Mbps) \\
\hline 31 & 13,4986 \\
\hline 91 & 12,2409 \\
\hline 171 & 12,0425 \\
\hline \hline RTS & 13,4213 \\
\hline 2346 & 13,4676 \\
\hline 2266 & 13,5286 \\
\hline 1823 & \\
\hline SLOTTIME & 12,8384 \\
\hline 2306 & 12,7054 \\
\hline 2266 & 12,7145 \\
\hline 2106 & \\
\hline
\end{tabular}

Tabla 3. Tasa transferencia Córdoba - Comfenalco El Bosque

\begin{tabular}{|l|c|}
\hline ACK & Tasa de Transferencia (Mbps) \\
\hline 21 & 3,0835 \\
\hline 131 & 2,7747 \\
\hline 331 & 2,6278 \\
\hline RTS & \\
\hline 1906 & 2,8456 \\
\hline 1866 & 2,5267 \\
\hline 1546 & 2,7558 \\
\hline SLOTTIME & \\
\hline 1906 & 2,8456 \\
\hline 1866 & 2,5267 \\
\hline 1546 & 2,7258 \\
\hline
\end{tabular}

Tabla 4. Tasa transferencia Tebaida - Barrio Bosques de Pinares

\begin{tabular}{|l|c|}
\hline ACK & Tasa de Transferencia (Mbps) \\
\hline 281 & 6,0103 \\
\hline 321 & 6,4846 \\
\hline 351 & 6,5355 \\
\hline RTS & \\
\hline 2346 & 6,0110 \\
\hline 2266 & 5,8685 \\
\hline 2226 & 6,0357 \\
\hline SLOTTIME & \\
\hline 2346 & 6,0110 \\
\hline 2226 & 6,0357 \\
\hline 2186 & 5,7802 \\
\hline
\end{tabular}

esto, se tienen en cuenta sólo los puntos por encima de esta media para contabilizarlos y sumarlos, con el valor obtenido se definen intervalos con los cuales se crean los histogramas y por medio de la tendencia de éstos se busca la distribución de probabilidad que se ajuste con mayor confiabilidad.

- Para el ajuste del parámetro ACK TimeOut se observa que resulta significativo en cuanto al desempeño puesto que su variación puede producir los puntos más críticos del enlace. La media para esta variable es de aproximadamente 286, con una desviación estándar de 49,0713

- El parámetro RTS registra ciertos puntos críticos en el proceso de variación del rango de posibles valores, pero, en general mantiene una tasa de transmisión de información con tendencia a estar sobre la media en casi todo el rango. La media para esta variable es de aproximadamente 1823, con una desviación estándar de 301,9536.

- Para el ajuste del parámetro SLOTTIME, se presenta una dependencia con la variable que permite modificar la interfaz de usuario de AirOS, denominada umbral de fragmentación, en otras palabras, debido a que la función de fragmentación de la capa MAC que permite que una estación divida los paquetes de datos en tramas más pequeñas para evitar la necesidad de retransmitir tramas grandes en un ambiente de interferencias de radiofrecuencia; además de los tiempos requeridos para la sincronización de la comunicación, el tamaño de trama tiene asociado un tiempo de envío o Slot Time que, como es claro, varía dependiendo de dicho tamaño de trama. Lo que se debe tener en cuenta es que el slot time es más importante para la prevención de colisiones cuando varias estaciones están conectadas, en este caso,

Teniendo en cuenta los resultados del análisis reali- 
Tabla 5. Ejemplo de la tabla para el análisis de Kolmogorov Smirnov

\begin{tabular}{|c|c|c|c|c|c|}
\hline INTÉRVALO & FO & FOA & POA & PEA & PEA-POA \\
\hline 21 a 56,1 & 7 & 7 & 0,0814 & 0,1003 & 0,0189 \\
\hline 56,1 a 91,2 & 5 & 12 & 0,1395 & 0,2006 & 0,0610 \\
\hline 91,2 a 126,3 & 5 & 17 & 0,1977 & 0,3009 & 0,1032 \\
\hline 126,3 a 161,4 & 9 & 26 & 0,3023 & 0,4011 & 0,0988 \\
\hline 161,4 a 196,5 & 6 & 32 & 0,3721 & 0,5014 & 0,1293 \\
\hline 196,5 a 231,6 & 11 & 43 & 0,5000 & 0,6017 & 0,1017 \\
\hline 231,6 a 266,7 & 10 & 53 & 0,6163 & 0,7020 & 0,0857 \\
\hline 266,1 a 301,8 & 11 & 64 & 0,7442 & 0,8023 & 0,0581 \\
\hline 301,8 a 336,9 & 10 & 74 & 0,8605 & 0,9026 & 0,0421 \\
\hline 336,9 a 371,0 & 12 & 86 & 1 & 1 & 0 \\
\hline Total & $\mathbf{8 6}$ & & & & \\
\hline
\end{tabular}

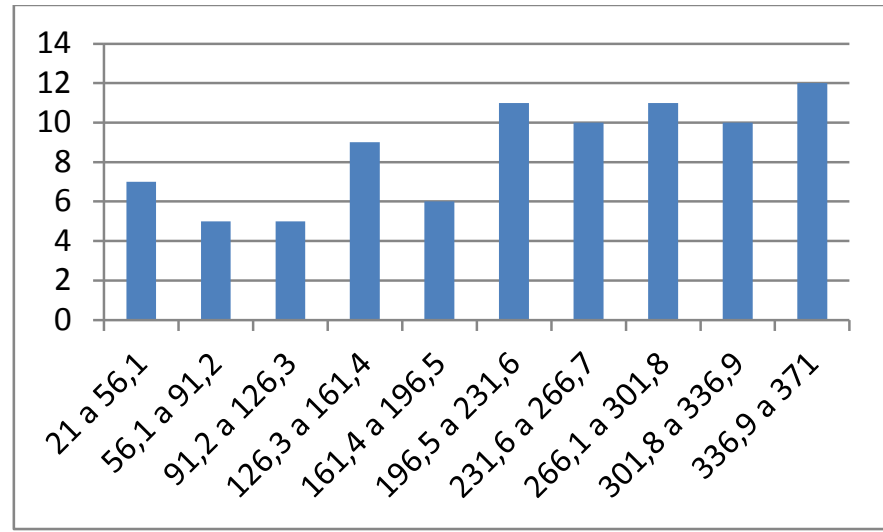

Figura 7. Ejemplo de la clase de histogramas obtenidos durante la investigación al cual se le ajusta la distribución de probabilidad continua uniforme con una confiabilidad del 95\%, debido a que el valor PEA-POA es menor que el valor denotado en la tabla de ajuste de Kolmogorov Smirnov

para la comunicación punto a punto. es evidente, según los datos registrados (6), que es posible conseguir una velocidad de transmisión superior a la media en la mayoría de los valores asignados a este parámetro; la parte de la ráfaga que contiene los datos (información útil) no superó el time slot, puesto que en ese caso se produciría colisión entre datos de distintos terminales y se perderían ambos paquetes. Con ello se obtuvo que la media para el umbral de fragmentación es de aproximadamente 1726 bytes, con una desviación estándar de 357,9567 bytes y que el slot time es $9 \mu \mathrm{s}$ para $802.11 \mathrm{~g}$ sin tener en cuenta los tiempos de establecimiento de la comunicación.

zado, se tiene entonces que la distribución que mejor puede describir la tendencia de los datos muestreados es la uniforme, por tanto, utilizamos la fórmula de la "media aritmética" especificada por la distribución continua uniforme, que puede interpretarse como la cantidad total de la variable distribuida en partes iguales entre cada observación y es definida como el valor característico de una serie de datos cuantitativos. (7)

Debido a que, la investigación tiene como fin encontrar los valores de ACK Time Out, RTS Time Out y Slottime donde exista máxima transferencia de información, se hace uso de la desviación estándar, la cual en un conjunto de datos (valores de ACK, RTS y Slot Time) es una medida de dispersión, que indica cuánto pueden alejarse los valores respecto al promedio (media), por lo tanto es útil para buscar probabilidades de que un evento ocurra, en este caso, que exista la tasa máxima de envío de datos, o, determinar entre qué rango de valores específicos de los parámetros estu- 
diados puede moverse la tasa esperada. (8)

Dicho lo anterior, se dice entonces que, los valores para ACK Time Out, RTS Time Out y Slot Time donde puede obtenerse la mayor tasa de velocidad de datos, va a encontrarse en el intervalo constituído por la media aritmética \pm la desviación estándar; para encontrar el valor específico de los parámetros estudiados, se plantea para trabajos futuros, realizar un modelo matemático donde puedan realizarse una aproximación más acertada de los valores y no tenga que realizarse este dispendioso trabajo de manera empírica.

\section{CONCLUSIONES}

Cabe destacar que con el procesamiento a través de Kolmogorov Smirnov se obtiene una varianza elevada, por ello, para posibles futuras aplicaciones de los datos obtenidos en este trabajo sería más práctico aplicar métodos de reducción de varianza y así conseguir un rango más limitado de valores óptimos para cada parámetro.

Las condiciones climáticas se registraron a lo largo del proceso de medición, pero para destacar si influyen de manera significativa en el comportamiento de cada parámetro, se hace necesario realizar un estudio detallado adicional al presente y de ese modo verificar dicha dependencia.

Se ha mostrado que resulta factible emplear enlaces inalámbricos para distancias largas, especialmente en zonas rurales; esto, teniendo en cuenta que se requiere el ajuste de algunos parámetros experimentando las posibilidades que brindan o permiten los estándares de muchos dispositivos accesibles en el mercado actual.

Debe tenerse claro que las distancias alcanzadas sin que se deteriore la calidad del enlace son restringidas debido a las limitaciones regulatorias y además las propias limitaciones del estándar utilizado, por esto, es vital modificar de manera adecuada el parámetro ACK Time Out.

Para el primer escenario planteado con anterioridad se tiene la distancia más corta en comparación con los demás enlaces punto a punto; por tal motivo se puede observar que es imperceptible la afectación por posibles retransmisiones, la mayoría de paquetes llegan a su destino en el primer intento debido a que la probabilidad de colisión es muy baja, esta situación se hace un poco desfavorable a medida que aumenta la distancia en los demás enlaces.

\section{AGRADECIMIENTOS}

Los autores agradecen a la Universidad del Quindío, la Vicerrectoría de Investigaciones, el programa de Ingeniería Electrónica y el grupo de investigación en telecomunicaciones (GITUQ) por su apoyo.

Este artículo es el resultado de un trabajo de grado, el cual a su vez hizo parte del proyecto de investigación "MODELO PARA LA ESTIMACIÓN DE PARÁMETROS ACK TIME OUT, CTS TIMEOUT Y SLOTTIME PARA ENLACES DE LARGA DISTANCIA 802.11g." con código 599, financiado por la Vicerrectoría de Investigaciones de la Universidad del Quindío.

\section{BIBLIOGRAFÍA}

1. Wheat J. 2001. Designing a wireless network. Estados Unidos: Syngress Publishing, Inc.

2. Jara, P. Nazar, P. (2009) "Estándar IEEE 802.11 X de las WLAN". Departamento de Ingeniería en sistemas de información Facultad Regional Tucumán Universidad Tecnológica Nacional - U.T.N edUTecNe, 1, 9-11. Consultado Septiembre 7, 2013, en http://www.edutecne.utn.edu.ar/monografias/standard_802_11.pdf.

3. IEEE-SA (2006). "Part 11: Wireless LAN Medium Access Control (MAC) and Physical Layer (PHY) specification". En IEEE (Society). Draft Standard for Information Technology: Telecommunications and information exchange between systems Local and metropolitan area networks (59-80). New York: Three Park Avenue

4. De la Mora, E. M. 2002. Metodología de la investigación. Desarrollo de la inteligencia. México: International Thomson Editores.

5. Astaiza, E, Bermúdez, H. F. y Gutiérrez, P. A. 2007. Simulación De Sistemas De Telecomunicaciones, Armenia: Ed Padilla Bejarano, Ferney.

6. Gráficas Medidas nuevas. (2014, Enero 10). Consultado Enero 23, 2014, en https://www.dropbox.com/sh/ zy58eem8sw9dywz/SOVrs2NsyW

7. Jirón, Q. (1998). "Nueva Enciclopedia autodidáctica -Física, Química y Matemáticas”, Colombia: Editorial LEXUS.

8. Triola, M. F. (2004), Estadística. Consultado Octubre 12, 2013, en http://books.google.com.co/books ? id $=$ Lj5VlatlLhsC\&pg $=$ PA75\&lpg $=$ PA 75\&dq $=$ de sviaci $\% \mathrm{C} 3 \% \mathrm{~B} 3 \mathrm{n}+$ estandar+libros\&source $=$ bl\&ots $=\mathrm{cMpEwJY0bO \& sig=2zf-j \_ UgK1Bj1}{ }_{-} \mathrm{lbMxoGjJ}$ gyqI\&hl=es\&sa=X\&ei=J9rhUqqDEfe_sQT02YKYD

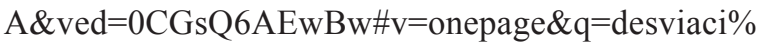
$\mathrm{C} 3 \% \mathrm{~B} 3 \mathrm{n} \% 20$ estandar\%20libros\& $\mathrm{f}=$ false. 